\title{
Short period fast waves in solar coronal loops
}

\author{
F. C. Cooper ${ }^{1}$, V. M. Nakariakov ${ }^{1}$, and D. R. Williams ${ }^{2}$ \\ 1 Physics Department, University of Warwick, Coventry, CV4 7AL, UK \\ e-mail: cooperf@astro.warwick.ac.uk \\ 2 MSSL, University College London, Dorking, Surrey, RH5 6NT, UK \\ e-mail: drw@mssl.ucl.ac.uk
}

Received 4 June 2003 / Accepted 7 July 2003

\begin{abstract}
Short period fast magnetoacoustic waves propagating along solar coronal loops, perturbing the loop boundary along the line of sight (LOS), may be observed by imaging telescopes. The relationship between the difference in emission intensity, the angle between the LOS and the direction of propagation and the wave amplitude and wavelength, is explored for kink and sausage fast waves. It is shown that the compressibility of the plasma in the loop significantly affects the observability of the waves. For both wave types there is an optimal observation angle which is determined by the ratio of the wave length and the loop radius. The change of the observational conditions because of the loop curvature predicts a significant, up to an order of magnitude, change in the observed wave amplitude. This prediction is confirmed by the analysis of the evolution of the fast wave train amplitude, observed with the SECIS instrument. The wave train amplitude experiences a sharp increase and then a decrease along the loop. The observational results are in a good agreement with the theory.
\end{abstract}

Key words. magnetohydrodynamics (MHD) - waves - Sun: activity - Sun: corona - Sun: oscillations

\section{Introduction}

Fast magnetoacoustic waves may be guided by field-aligned regions of enhanced plasma density corresponding to regions of decreased Alfvén speed (Edwin \& Roberts 1982, 1983, 1988). It was found that the waves are highly dispersive in the long wavelength part of the spectrum and that all modes except the kink, have a lower cut-off wave number. The high dispersion of the waves may lead to formation of a characteristic signature of the signal observed in a coronal loop, this was applied to interpretation of coronal radio-pulsations (Roberts et al. 1983, 1984). Later on, Nakariakov \& Roberts (1995) established that the qualitative dispersive properties depend weakly upon the specific profile of the density. However, one important exception was found: there is a certain critical steepness of the density profile which separates two distinct scenarios of the wave evolution. If the density profile is steep enough, the derivative of the wave group speed with respect to the frequency changes its sign, and consequently the group speed has a minimum at a certain value of the wave number. The presence of such a minimum is responsible for the formation of the characteristic signature of the waves. If the density profile is smoother than that critical value, the group speed decreases gradually with the wave number. The group speed dependence on the wave number also has important implications for nonlinear phenomena, in particular, the modulational instability (Nakariakov et al. 1997). These days the actual steepness of

Send offprint requests to: V. Nakariakov, e-mail: valery@astro.warwick.ac.uk density profiles in the loops is a subject of an intensive discussion in connection with interpretation of the quick decay of coronal loop kink oscillations (Nakariakov et al. 1999; Ofman \& Aschwanden 2002; Goossens et al. 2002). Unfortunately, the presently available resolution of coronal telescopes (about $1 \mathrm{Mm}$ or worse) can be about the radius of the loop or even larger, which makes impossible the direct determination of the density profile.

Fast magnetoacoustic waves propagating along coronal loops may provide us with a tool for the determination of physical parameters of the loops. In particular, dispersive evolution of the waves with wavelengths comparable with the loop tube radius contains information about the steepness of the density profile. It may be possible to estimate the density profile through temporal characteristics of the wave. The presently achievable temporal resolution (less then a second) is much higher than the spatial resolution, from the point of view of detection of fast waves. Indeed, a fast wave of a $1 \mathrm{~s}$ period and of an estimated speed of about $1 \mathrm{Mm} \mathrm{s}^{-1}$ has a wavelength of about $1 \mathrm{Mm}$, so it can be resolved in time but not in space. Observationally, there is overwhelming evidence of short period coronal pulsations detected in the radio band, associated with fast magnetoacoustic waves (e.g. Roberts et al. 1983, 1984; Aschwanden 1987). Very recently, quasi-periodic disturbances propagating along a coronal loop were observed in visible light using the imaging SECIS instrument (Phillips et al. 2000) during the 1999 August 11th solar eclipse (Williams et al. 2001, 2002). The detected mean period was about $6 \mathrm{~s}$ 


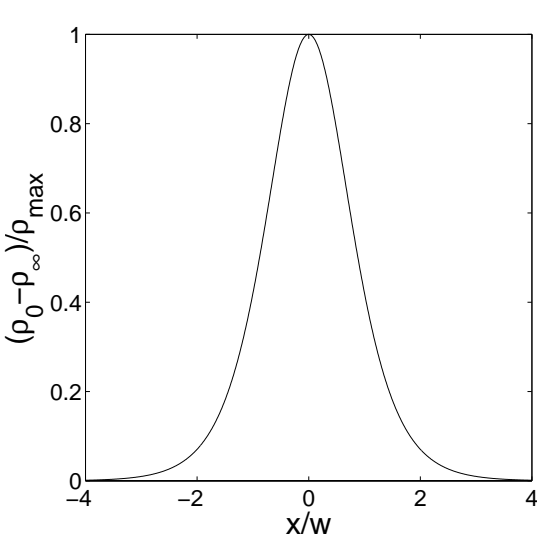

Fig. 1. The symmetric Epstein profile representing the plasma density across the loop, normalized to the loop width parameter $w$ and the maximum plasma density $\rho_{\max }$.

and the speed was estimated as $2.1 \mathrm{Mm} \mathrm{s}^{-1}$, suggesting the interpretation in terms of fast magnetoacoustic waves.

The lack of the resolution required complicates the direct application of well-developed MHD wave theory to interpretation of observed phenomena. Thus, adaptation of MHD wave theory to the reality of coronal observations becomes an important task. Such studies have been performed on observability of various types of coronal waves with spectral instruments (e.g. Erdelyi et al. 1998; Sakauri et al. 2002; Zaqarashvili 2003). As wavelengths of coronal propagating disturbances, with frequencies of the order of a second, are of the same order as the loop cross-section diameter, the short wavelength effects on the wave observability should be investigated. Recently, Cooper et al. (2003) (Paper I) found theoretically that almost incompressible short wavelength kink magnetoacoustic modes of cylindrical plasma structures can be detected with imaging telescopes as propagating disturbances of emission intensity. This effect is connected with modulations of the observed column depth of the loop by the kink perturbation. An important characteristic of the effect is that the intensity variation detected is dependent upon the angle between the direction of wave propagation and the line-of-sight (LOS). In Paper I, the loop cross-section was modeled by a step density profile. It is interesting if the same effect arises in the case of a loop with a smooth density profile and if it may provide us with a diagnostic tool for determination of the width of this profile.

The aim of this paper is to generalize the study performed in Paper I, taking into account effects of plasma compressibility, and demonstrate the applicability and importance of these results to the interpretation of short period coronal waves, in particular, to SECIS project results.

\section{The model}

We model a coronal loop as a magnetic slab with a smooth density profile, given by the profile function

$\rho_{0}=\rho_{\max } \operatorname{sech}^{2}\left(\frac{x}{w}\right)+\rho_{\infty}$

where $\rho_{\max }, \rho_{\infty}$ and $w$ are constant. Here, the parameter $\rho_{\max }$ is the density at the center of the inhomogeneity, $\rho_{\infty}$ is the density at $x=\infty$ and $w$ is a parameter governing the inhomogeneity width. This inhomogeneity, plotted in Fig. 1, is called the symmetric Epstein profile (see, e.g. Adams 1981; Nakariakov \& Roberts 1995). The plasma is inhomogeneous across the straight and uniform magnetic field $\boldsymbol{B}_{0}=B_{0} \hat{z}$. In the zero- $\beta$ limit considered, the equilibrium total pressure balance is fulfilled identically.

According to Nakariakov \& Roberts (1995), linear perturbations of the transversal plasma velocity $V_{x}=$ $U(x) \exp (i \omega t-i k z)$ are described by the equation

$\frac{\mathrm{d}^{2} U}{\mathrm{~d} x^{2}}+\left[\frac{\omega^{2}}{C_{A \infty}^{2}}-k^{2}+\frac{\omega^{2}}{C_{A \max }^{2}} \operatorname{sech}^{2}\left(\frac{x}{w}\right)\right] U=0$,

where $C_{A \infty}$ is the Alfvén speed as $x \rightarrow \infty$ and $C_{A \max }=$ $B_{0} / \sqrt{4 \pi\left(\rho_{0}-\rho_{\infty}\right)}$ is an Alfvén speed based upon the difference in density between that at $x=0$ and as $x \rightarrow \infty$. As the corresponding profile of the Alfvén speed has a minimum at the center of the slab, the slab is a refractive waveguide for fast magnetoacoustic waves (see Edwin \& Roberts 1988 for discussion).

The eigenvalue problem stated by Eq. (2) supplemented by the boundary conditions $U(x \rightarrow \pm \infty) \rightarrow 0$ can be solved analytically, (see, e.g., Landau \& Lifshitz 1958; Adams 1981). The eigenfunctions describing kink and sausage modes are respectively given by

$U=\operatorname{sech}^{v}(x / w), \quad v=\frac{|k| w}{C_{A \infty}} \sqrt{C_{A \infty}^{2}-a^{2}}$

and

$U=\frac{\sinh (x / w)}{\cosh ^{\lambda}(x / w)}, \quad \lambda=\frac{|k| w}{C_{A \infty}} \sqrt{C_{A \infty}^{2}-a^{2}}+1$,

where $a=\omega / k$ is the phase speed. The phase speed is determined by the dispersion relations

$\sqrt{C_{A \infty}^{2}-a^{2}}=|k| w \frac{C_{A \infty}}{C_{A 0}^{2}}\left(a^{2}-C_{A 0}^{2}\right)$

and

$\frac{|k| w}{C_{A 0}^{2}}\left(a^{2}-C_{A 0}^{2}\right)-\frac{2}{|k| w}=\frac{3}{C_{A \infty}} \sqrt{C_{A \infty}^{2}-a^{2}}$,

for the kink and the sausage modes, respectfully. Here $C_{A 0}=$ $C_{A \infty} C_{A \max } /\left(C_{A \infty}^{2}+C_{A \max }^{2}\right)^{1 / 2}$ is the Alfvén velocity at the center of the profile, $x=0$. These solutions are well-known in quantum mechanics (e.g. Landau \& Lifshitz 1958) and in the theory of optical fibrils (Adams 1981). In the coronal context, profile (1) and the kink mode solution given by Eqs. (3) and (5) have been introduced by Nakariakov \& Roberts (1995). The sausage mode solution given by Eqs. (4) and (3) has not been used in the coronal context yet.

For the purposes of this study, solutions (3) and (4) have one important advantage: the same function describes the perturbation of the plasma inside and outside the slab. The knowledge of the transverse component of the velocity allows us to determine the density perturbation. From the continuity equation we get

$\tilde{\rho}=-\int \frac{\partial\left(\rho_{0} \tilde{V}_{x}\right)}{\partial x} \mathrm{~d} t$ 

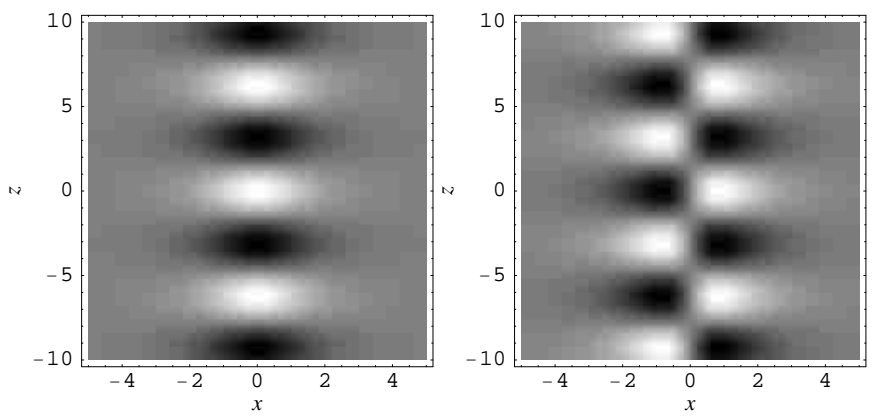

Fig. 2. Loop segment plasma velocity profiles for kink (left) and sausage (right) modes. Light indicates velocity in the positive $x$ direction, dark indicates velocity in the negative $x$ direction and grey indicates zero velocity.
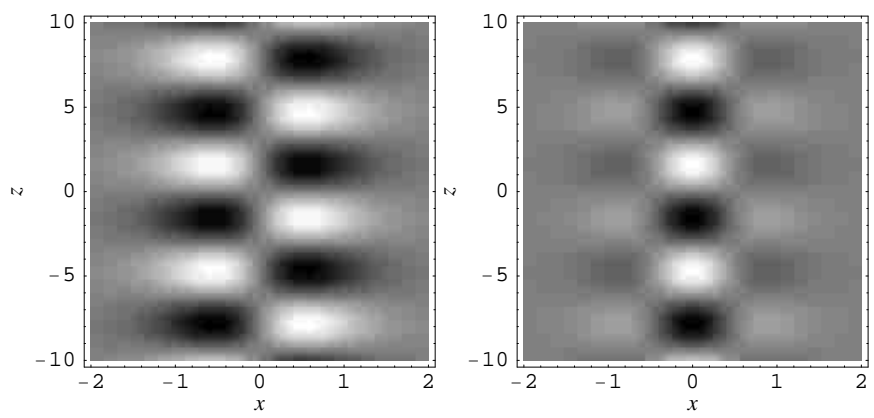

Fig. 3. Loop segment density perturbation profiles for kink, (left) and sausage (right) modes. Light indicates positive density perturbations, dark indicates negative density perturbations i.e. a decrease of the unperturbed loop density. Grey indicates low or little perturbation.

where $\rho_{0}$ is the equilibrium according to Eq. (1) and the tilde represents a small perturbation. We then have expressions for the total density across the loop, including the perturbations. These are plotted in Fig. 3.

\section{Observability of MHD modes with imaging telescopes}

Both kink and sausage modes produce modulation of the intensity of emission generated in the plasma and, consequently, can be observed with imaging telescopes. The intensity of emission generated in the optically thin coronal plasma is proportional to the line integral of the electron number density squared, along the LOS,

$I \propto \int_{(\operatorname{LOS})}\left[\rho_{0}(l)+\tilde{\rho}(l)\right]^{2} \mathrm{~d} l$.

Here $l$ is the coordinate along the LOS.

Following Paper I, we consider a straight segment of a coronal loop, parallel to the $z$-axis and having an angle $\theta$ with the LOS. In the plane $(x z)$ formed by the loop segment and the direction of the inhomogeneity, the equation of the LOS is given by

$z=x \cot \theta+p / \sin \theta$,

where $p$ represents the coordinate across the LOS and consequently along the image. Equation (9) states the path of the integration in Eq. (8).
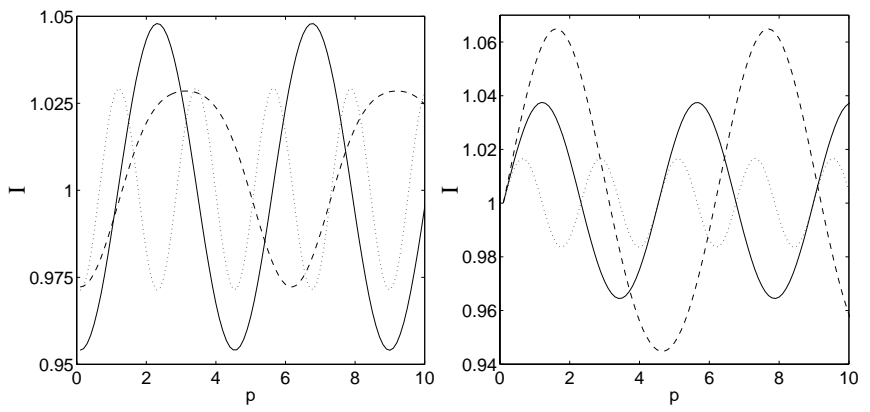

Fig. 4. Example intensities $I$ as a function of image distance, normalized to the unperturbed intensity and loop width for kink (left) and sausage (right) perturbations. The solid lines are given by the parameters, amplitude $a=0.05$, wave number $k=1$, angle between the loop axis and the $\operatorname{LOS} \theta=45^{\circ}$, the dashed by $a=0.1, k=1, \theta=75^{\circ}$ and the dotted by $a=0.02, k=2, \theta=45^{\circ}$.

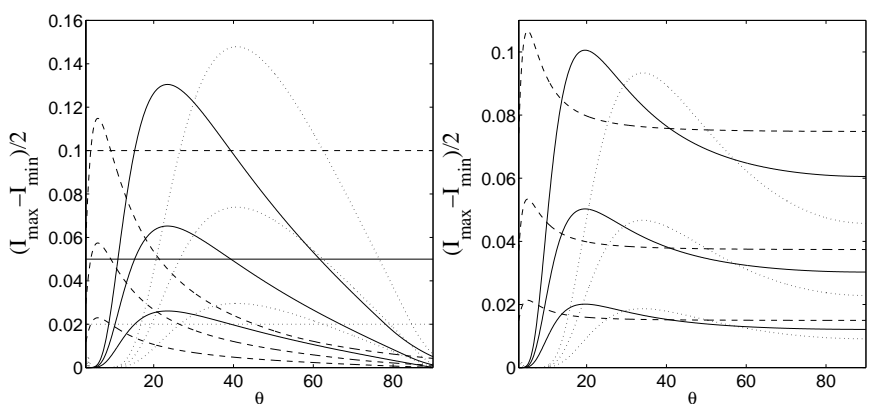

Fig. 5. Dependence of the observed amplitude of emission intensity variation along a straight segment of a coronal loop in the presence of a harmonic kink (left) and sausage (right) perturbation upon the angle between the loop axis and the LOS, $\theta$. Here the wave number $k=0.2$, dashed, 0.9, solid and 2, dotted with the amplitudes $a=0.1$, top, 0.05 , middle and 0.02 , bottom curves. The straight line in the kink case indicates if the wave amplitude appears larger, above or smaller, below than the actual perturbation amplitude. Only a small region is amplified in the sausage case so no lines are drawn.

We evaluate integral (8) numerically for the background density given by Eq. (1) and the perturbations by Eqs. (3) and (4) for kink and sausage modes, respectively. Examples of the snapshots of the observed intensity are shown in Fig. 4, where the wave number $k$ is normalized to the reciprocal of the width parameter $1 / w$, defined in Eq. (1) and Fig. 1. The observed intensity depends upon several parameters: the ratio of the perturbation wavelength to the characteristic width of the loop $w$, the LOS angle $\theta$ and the relative amplitude of the displacement of the loop effective boundary. Parametric studies of the observed emission intensity are shown in Figs. 4-7. (There the intensity variation amplitude is determined as the difference between the maximum and minimum of each intensity curve.)

From each graph in Fig. 5 it is clear that there is an optimal angle $\theta_{\max }$ for the observation of both kink and sausage modes. This optimal angle is plotted against $k$ in Fig. 7. The results for kink modes are not qualitatively different from the incompressible case studied in Paper I. The left panel of Fig. 5 shows that the kink modes can be observed with an imaging telescope only if the LOS angle is somewhere between, but not at, zero and ninety degrees. The perturbation amplitude does 

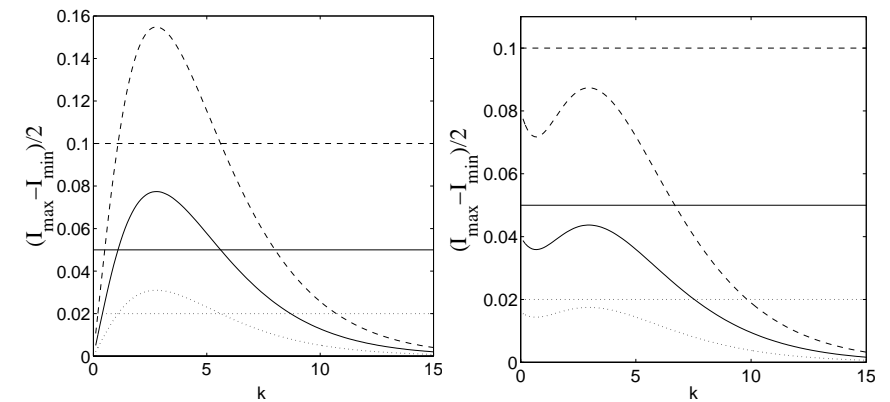

Fig. 6. Dependence of the observed amplitude of emission intensity variation along a straight segment of a coronal loop in the presence of a harmonic kink (left) and sausage (right) perturbation upon the wave number $k$. Here the angle between the loop axis and the $\operatorname{LOS} \theta=45^{\circ}$ and the amplitude $a=0.1$, dashed, 0.05 , solid and 0.02 , dotted.

not seem to affect significantly the position of the maximum. (However, higher amplitudes can actually change the position of the maximum because of nonlinear effects which are not accounted here). The observed intensity amplitude grows with the wave number to a maximum and then decreases (the left panel of Fig. 6). The optimal angle also grows with the normalized wave number for $0.1<k<2$ (Fig. 7).

In a loop with a smooth profile, the sausage mode has an optimal observation angle $\theta<90^{\circ}$ which may be much less then the incompressible sausage mode optimal angle, $90^{\circ}$. The physical reason for this qualitative discrepancy is connected with the fact that in the case of a smooth plasma density profile, the sausage modes are essentially compressible. Indeed, the right panel of Fig. 3 demonstrates that the density perturbation is in anti-phase with respect to the internal and external parts of the profile. Obviously, the optimal angle of observation corresponds to the case when the LOS passes either through the external and internal maxima or minima in the density perturbation (e.g., about $45^{\circ}$ for the right panel of Fig. 3), giving the maximum intensity contrast. In the incompressible case considered in Paper I, the optimal angle for the sausage mode was $90^{\circ}$ exactly. The right panel of Fig. 5 shows that the sausage mode optimal observation angle is somewhere between, but not at, zero and ninety degrees. However, in contrast to the case of kink modes which are difficult to observe when the LOS is perpendicular to the loop segment axis, sausage waves can be seen. As in the case of the kink mode, the optimal angle is not significantly affected by the perturbation amplitude. There is a least favorable wave number for low $k$ and after a maximum as the wave number increases the observed amplitude decreases (the right panel of Fig. 6), but the minimal observability is not well pronounced. The optimal angle grows with the wave number (Fig. 7).

\section{Effects of loop curvature}

Since the observability of fast magnetoacoustic waves is determined by the angle between the LOS and the loop segment, it is important to account for the effects of loop curvature. The change of the LOS angle would lead to variation of the observed amplitude of the wave. This can cause amplification or attenuation of the observed wave amplitude, which should not

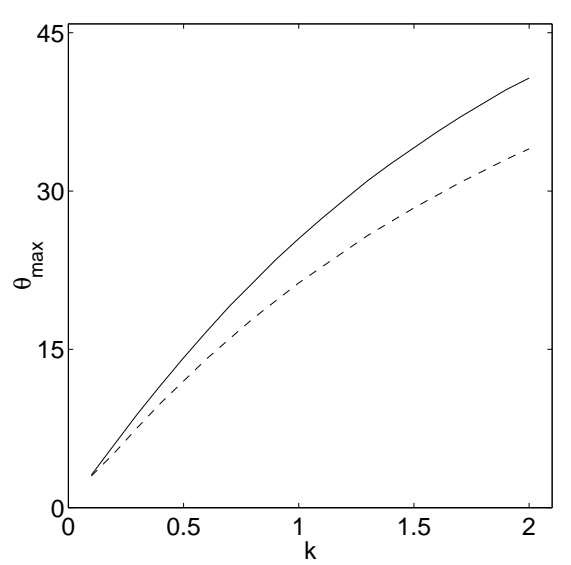

Fig. 7. Dependence of the optimal observation angle along a straight segment of a coronal loop in the presence of a harmonic kink, solid, and sausage, dashed, perturbation upon the wave number $k$. To graphical accuracy the lines for the amplitudes $a=0.1,0.05$ and 0.02 fall on top of one another.

be confused with the actual growth or decrease of the amplitude (e.g., the growth connected with the stratification, Ofman et al. 1999).

When the wavelength is comparable with the loop crosssection width and is much shorter than the radius of curvature of the loop, the loop curvature does not affect the fast wave propagation. Consequently, we may study the curvature effects on the wave observability using the results of Sect. 3 . Consider a semi-circular loop. The loop plane has an angle $\sigma$ with the LOS, Fig. 9. For simplicity, we assume that the LOS is in the plane of the wave polarization. The angle between the LOS and the tube axis $\theta$ for any segment is then given by

$\theta=\arccos (\cos \phi \sin \alpha-\sin \phi \cos \alpha)$,

where $\alpha$ is the angle between the solar surface and the LOS and $\phi$ is the angle between a loop radial line to the observed point and the solar surface, Fig. 8. The observed amplitude as a function of observation angle for various parameters is shown in Fig. 10. The form of the function plotted in Fig. 5 but modified to the loop semi circle is retained, reflected about the point where the angle between the loop and the LOS is $90^{\circ}$ and $0^{\circ}$. One can clearly see that the observed intensity is strongly modified along the loop profile despite the lack of any real wave amplification or attenuation.

\section{Application to SECIS observations}

This result has applications to the apparent impulsive, fastmode wave presented by Williams et al. (2001). In the companion paper, Williams et al. (2002) analyze the phase and amplitude progression of an oscillatory signal, with a period of several seconds, along a coronal loop apex observed in Fe XIV $\lambda 5303 \AA$ by SECIS. Active region NOAA 8651 appeared on the north-west limb of the Sun on the day of eclipse (1999-Aug.-11), and the loop in question ostensibly straddled the limb, much as the example in Fig. 9 (left panel). Adopting a semi-circular geometry for the loop implies that the observer 


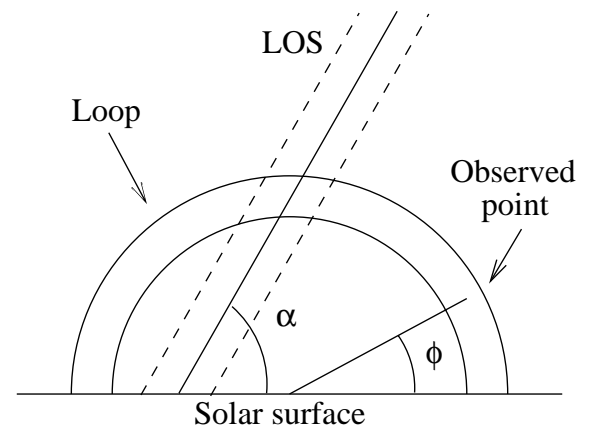

Fig. 8. A diagram of the loop geometry used. The angle $\alpha$ is the angle between the LOS and the solar surface and $\phi$ is the angle between a loop radial line to the observed point and the solar surface. We then use Eq. (10) to find the angle between the loop segment axis and the LOS, $\theta$.
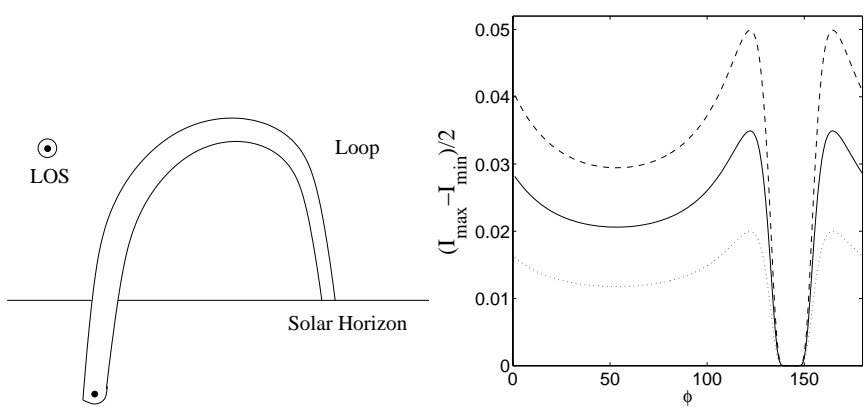

Fig. 9. A Typical observation of an off limb coronal loop, left. The loop plane has the angle $\sigma$ to the LOS. Changing this angle, right, has the effect of modifying the amplitude of the observed signal by $\cos \sigma$ assuming that the LOS is in the plane of the wave polarization. The dotted line corresponds to $\cos \sigma=0.4$, the solid to 0.7 and the dashed to 1 .

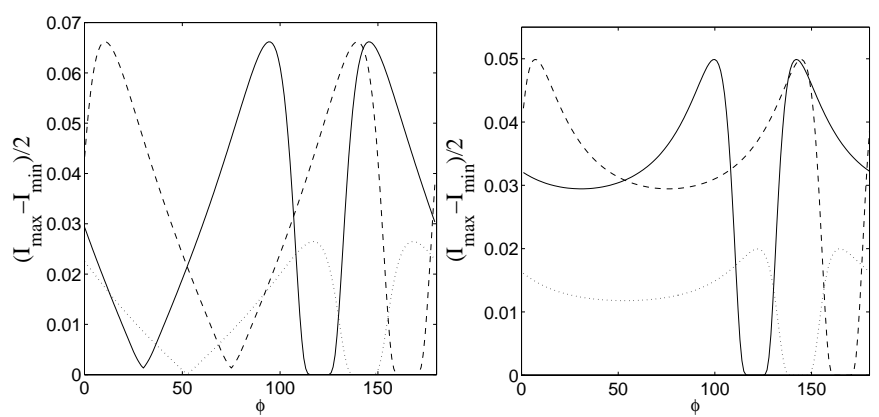

Fig. 10. Dependence of the observed amplitude of emission intensity variation along a coronal loop in the presence of a harmonic kink (left) and sausage (right) perturbation upon the position on the loop at which the observation is made. Here the wave number $k=1$, with the amplitudes $a=0.05$, solid and dashed and 0.02, dotted. The angle $\phi$ is the angle between the tangent to the solar surface and the loop section observed where the angle between the solar surface and the LOS $\alpha=30^{\circ}$, solid, $52.5^{\circ}$, dotted and $75^{\circ}$, dashed.

views the loop at an angle of $\sigma \sim 15$ degrees. One can immediately recognize that, since $\cos \sigma \sim 1$, the effect of amplitude modification would be at (or near) its greatest.

The observationally determined wave length of the propagating disturbances is about $12 \mathrm{Mm}$ and the loop width is about 5-10 Mm, giving the normalized wave number $k$ of

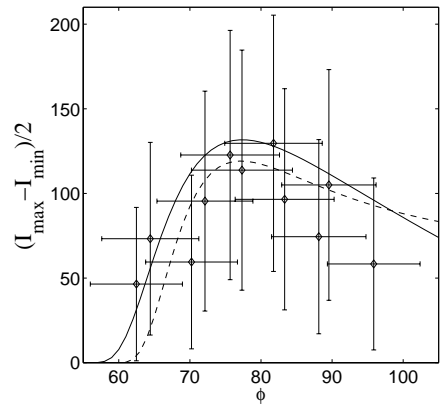

Fig. 11. A plot of the maximum amplitude at the most frequent scale of the points, analyzed by Williams et al. (2001), against their loop position. The $x$ axis error bars are the uncertainty in position and the $y$ axis error bars have been calculated statistically by taking the square root of the number of photons and normalizing with respect to time. The figure also includes possible lines as in Fig. 10 for kink, solid and sausage, dashed, modes with the parameters $a=0.05, k=1$ and $\alpha=51^{\circ}$, solid, and $55^{\circ}$, dashed.

about two or three. According to Fig. 6, observability of perturbations with wave numbers in this range is about optimal. Perhaps, this, together with optimal observation angle of the loop plane, explains the very fact of the successful detection of these propagating disturbances by SECIS. The theory developed above predicts that the variation of the LOS angle along the loop should lead to variation of the observed amplitude of the propagating disturbance. In particular, the observed wave amplitude should experience increases and decreases at different segments of the loop (Fig. 10). This motivated our study of the amplitude variation as a function of the position at the loop in the SECIS data.

Figure 11 demonstrates the amplitude of the wave moving through the SECIS points analyzed by Williams et al. (2001). The wave amplitudes at these points, calculated from the wavelet transform and are plotted against the estimated loop position. Note that the intensity used here is an amplitude and not an absolute intensity. The horizontal axis error bars represent the uncertainty in position and the vertical axis error bars were calculated statistically by taking the square root of the number of photons per data point and normalizing with respect to time $\left(\mathrm{s}^{-1}\right)$. Note that these values are estimates and not rigorously calculated experimental errors. One can clearly see a peak, roughly between 75 and 85 degrees along the loop and assuming the vertical axis errors are not significantly different, this feature may be explained by the LOS effect. Crudely judging by eye the kink mode at a LOS angle of $51^{\circ}$ seems to fit the best. However, as the sausage wave can exhibit similar behavior, the observational data do not allow us to determine which of the modes is responsible for the propagating disturbances observed. The analysis of the estimated propagation speed, of about $2100 \mathrm{~km} \mathrm{~s}^{-1}$, makes the sausage wave interpretation more favorable, as it is likely that this value of speed is likely to be closer to the Alfvén speed outside the loop. The size of the error bars does not allow us to make any conclusions about the observed wave dissipation. 


\section{Conclusions}

Both kink and sausage transverse compressible (or fast magnetoacoustic) wave modes of coronal loops, which displace the loop along the $L O S$, may be detected using imaging telescopes. This effect is pronounced for wave lengths comparable with the loop width, making it relevant for short period waves only. The observed intensity amplitude as a function of the wave parameters and observation angle behaves in a similar, but not identical way to an incompressible wave in a loop (Paper I). The main effect brought by the compressibility of the medium is the departure of the optimal observation angle of the sausage wave from 90 degrees.

The loop curvature changes the observational angle, affecting the observed wave amplitude. This leads to a modulation in a form of significant, up to an order of magnitude, increases and decreases of the observed amplitude along the loop. The effect is more pronounced for kink perturbations. As solar coronal observations usually show strong noise, the observational manifestation of the amplitude variation may look like an abrupt appearance and disappearance of the waves in certain segments of loops.

The analysis of the amplitude variation of propagating disturbances observed by SECIS reveals that the wave train amplitude experiences a significant increase and then decrease. It is difficult to quantify this phenomenon because of strong observational noise, but, qualitatively, the existence of the strong change of the amplitude is obvious. Observational detection of this phenomenon may be considered as an additional proof of the interpretation of the SECIS observed propagating disturbances as fast magnetoacoustic wave trains.

The dependence of the LOS effect discussed above upon the ratio of the wave length and the loop width, makes it potentially useful for MHD coronal seismology. However, further observational and theoretical studies are necessary.
Acknowledgements. FCC acknowledges financial support from PPARC.

\section{References}

Adams, M. J. 1981, An Introduction to Optical Waveguides (John Wiley \& Sons)

Aschwanden, M. J. 1987, Sol. Phys., 111, 113

Cooper, F. C., Nakariakov, V. M., \& Tsiklauri, D. 2003, A\&A, 395, 765

Edwin, P. M., \& Roberts, B. 1982, Sol. Phys., 76, 239

Edwin, P. M., \& Roberts, B. 1983, Sol. Phys., 88, 179

Edwin, P. M., \& Roberts, B. 1988, A\&A, 192, 343

Erdelyi, R., Doyle, J. G., Perez, M. E., \& Wilhelm, K. 1998, A\&A, 337,287

Goossens, M., Andries, J., \& Aschwanden, M. J. 2002, A\&A, 394, L39

Landau, L., \& Lifshitz, E. 1958, Quantum Mechanics (Pergamon Press)

Nakariakov, V. M., \& Roberts, B. 1995, Sol. Phys., 159, 399

Nakariakov, V. M., Ofman, L., DeLuca, E. E., Roberts, B., \& Davila, J. M. 1999, Science, 285, 862

Nakariakov, V. M., Roberts, B., \& Petrukhin, N. S. 1997, J. Plasma Phys., 58, 315

Ofman, L., \& Aschwanden, M. J. 2002, ApJ, 576, L153

Ofman, L., Nakariakov, V. M., \& DeForest, C. E. 1999, ApJ, 514, 441

Phillips, K. J. H., Read, P. D., Gallagher, P. T., et al. 2000, Sol. Phys., 193, 259

Roberts, B., Edwin, P. M., \& Benz, A. O. 1983, Nature, 305, 688

Roberts, B., Edwin, P. M., \& Benz, A. O. 1984, ApJ, 279, 857

Sakurai, T., Ichimoto, K., Raju, K. P., \& Singh, J. 2002, Sol. Phys., 209, 265

Williams, D. R., Phillips, K. J. H., Rudawy, P., et al. 2001, MNRAS, 326, 428-436

Williams, D. R., Mathioudakis, M., Gallagher, P. T., et al. 2002, MNRAS, 336, 747

Zaqarashvili, T. 2003, A\&A, 399, L15 